\title{
MANIFESTATIONS OF SOLAR MAGNETIC FIELDS
}

\author{
S.K. SOLANKI \\ Institute of Astronomy, ETH, CH-8092 Zürich, Switzerland
}

\begin{abstract}
The magnetism of the Sun manifests itself in innumerable ways, many of which constitute what is referred to as solar magnetic activity, while others are counted among the phenomena of the quiet Sun. After a brief overview of the structure of the solar magnetic field, a few examples of its manifestations are pointed out.
\end{abstract}

\section{Introduction}

The magnetic field of the Sun has observable consequences ranging from changes in $p$-mode frequencies to the rapid acceleration of parts of the solar wind to high speeds. The vast variety of manifestations is due to the highly complex and dynamic nature of solar magnetism. These properties in turn result from the interaction of the magnetic field with solar differential rotation, convection, radiation, oscillations and waves.

On a large scale the complex structure of the solar magnetic field manifests itself by the presence of bipolar active regions and the magnetic network in the quiet Sun. This complexity continues down to the smallest observable scales, corresponding to a fraction of an arc $s$, with the magnetic field being highly structured at all scales.

In the photosphere and interior of the Sun the magnetic field is concentrated into fux tubes or bundles of magnetic field lines. In the photosphere these flux tubes generally have field strengths of 1-3 kG and range in size from roughly $100 \mathrm{~km}$ (bright magnetic elements) to tens of thousands of $\mathrm{km}$ (dark sunspots). There is also some evidence for a weak, possibly turbulent magnetic field between the flux tubes. Although a considerable fraction of the magnetic flux may be in weak-field form, the flux tubes contain almost all the magnetic energy. Nevertheless, they cover only a very small fraction of the solar surface (on the order of $1 \%$ ). This implies that in the solar photosphere the magnetic field is highly filamented.

The magnetic flux tubes expand with height. This expansion is particularly rapid in the mid chromosphere, where the field becomes almost horizontal, forming a magnetic canopy, i.e. a region of field overlying a field-free atmosphere. Above this layer basically the whole of the solar atmosphere is permeated by a magnetic field. Still higher up, in the corona, we need to distinguish between "open" field lines (i.e. field lines that reach out into interplanetary space) and "closed" (such as those forming loops or arcades).

On the whole the field becomes progressively more homogeneous in strength with height, although it is by no means near complete homogeneity in the corona, in particular in active regions. Conversely, it becomes increasingly more inhomogeneous in direction. Whereas in the photosphere flux tubes are more or less vertical, almost any direction is possible in the corona and current sheets abound at tangential discontinuities of the field (at least according to theory).

In determining the magnitude and variety of ways in which the magnetic field manifests itself an important role is played by the approximate relative energy density of the field, $E_{B}=B^{2} / 8 \pi$, of the gas (thermal energy density), $E_{t}=\frac{3}{2} p$, and of the motions (kinetic energy density), $E_{k}=\rho v^{2} / 2$. Here $B$ is the magnetic field strength, $p$ is the gas pressure, $\rho$ is its density and $v$ its velocity.

The relative values of these 3 quantitites are a strong function of height. In the solar convection zone $E_{B} \approx E_{k}$ and $E_{B} \ll E_{t}$, so that manifestations due to the field are expected to be small. As we go up in height $E_{B}$ becomes increasingly important. Thus, in the photosphere $E_{B} \approx E_{t} \gg E_{k}$, 
in the chromosphere $E_{B}>E_{t} \gtrsim E_{k}$ and in the corona $E_{B} \gg E_{t}, E_{B} \gg E_{k}$. Finally, in the solar wind outside the Alfvén radius $E_{k}>E_{B}$, so that the influence of the magnetic field is expected to diminish again in these layers.

\section{Subsurface manifestations}

The main observable consequence of subsurface magnetic fields is changes in the power and frequency of $p$-modes. The frequency variations are quite subtle ( 1 part in 10000 , Libbrecht \& Woodard 1990, Elsworth et al. 1994), but the reductions in power can be considerable (up to a factor of 2-3, Tarbell et al. 1988). Particularly dramatic is the absorption of intermediate degree $p$-modes by sunspots (Braun et al. 1992, Bogdan et al. 1992). The observations suggest that it is the layers near the surface (where the sound speed is low) which mainly contribute to the above variations.

\section{Photospheric manifestations}

In the solar photosphere the presence of the magnetic field is marked by sunspots, faculae (both in active regions and the quiet Sun) and abnormal granulation, all of which influence the local brightness. The root cause is a combination of the inhibition of convection by the magnetic field, which lowers the temperature, and the channelling of radiative and probably MHD wave flux to the surface, which raises the temperature. Depending on which of these influences is stronger either a darkening or a brightening is obtained. The sum of the local changes in photospheric brightness due to magnetic features contributes significantly, if not dominantly to the observed variations of the total solar irradiance (Willson \& Hudson 1991, Spruit 1982, Foukal \& Lean 1988, Solanki \& Unruh 1997).

Another way in which magnetic fields influence photospheric radiation is through the Zeeman and Hanle effects. They change line shapes and polarize, repsectively depolarize the line radiation. This provides us with a powerful direct diagnostic of the magnetic field. Consequently, the photosphere is the layer in which we know the most about solar magnetism. Let me briefly touch on three examples.

A remarkable property of photospheric flux tubes is that the observed field strength averaged over the whole magnetic feature remains unchanged to within $50 \%$ (between 1000 and $1500 \mathrm{G}$ in the mid photosphere) over 5-6 orders of magnitude in magnetic flux (Solanki \& Schmidt 1993), although for the smallest magnetic features (intranetwork elements) the field strength does decrease to a few $100 \mathrm{G}$ (Keller et al. 1994, Lin 1995, Solanki et al. 1996). This dependence of magnetic field strength on flux per magnetic element is well reproduced by convective collapse models of the formation of magnetic elements (Venkatakrishnan 1986).

Using the Zeeman effect it is straightforward to make 2-D maps of the solar magnetic field, i.e. magnetograms. Time series of magnetograms reveal an incessant emergence and cancellation of magnetic flux, as well as its constant motion on the solar surface (Martin 1988). Estimates of the flux emergence rate indicate that most flux emerges at small scales. The averaged flux emergence rates are: $10^{20} \mathrm{Mx}$ per day in active regions, $10^{22} \mathrm{Mx}$ per day in the form of ephemeral active regions and $10^{24} \mathrm{Mx}$ per day in the form of intranetwork fields (e.g. Harvey et al. 1975, Zirin 1987), which leads to a renewal of most of the quiet Sun flux on short time scales.

The constant changes in the surface magnetic field imply that reconnection between field lines is common, and may have a bearing on the coronal heating problem (Parker 1988, Title 1997).

The final example concerns the magnetic structure of sunspot penumbrae. Observations have shown that at least 2 magnetic components are present, which differ in inclination by $20-40^{\circ}$ and are structured at small scales (Degenhardt \& Wiehr 1991, Title et al. 1993). This unexpectedly complex magnetic structure provides an example of the types of tangential discontinuities expected according to theory in the upper atmosphere.

\section{Chromospheric and transition region manifestations}

In the chromosphere and the transition region the magnetic field begins to dominate energetically over the gas and plays a correspondingly important role in determining chromospheric structure and energetics (a role it shares with waves and radiation). Outside magnetic features the lower 
chromosphere may well be much cooler than standard models (Fontenla et al. 1993) suggest (Solanki et al. 1994, Carlsson \& Stein 1995).

There is a clear if often non-linear relationship between emission in chromospheric and transition region lines sensitive to temperature (such as Ca II H \& K or C IV) and the amount of magnetic flux measured in the underlying photosphere (e.g., Schrijver et al. 1989).

Other examples of chromospheric magnetic manifestations are prominences, spicules and explosive events. In the case of prominences the magnetic field is required to support dense material at coronal heights and to thermally insulate it against the surrounding hot coronal gas. The Hanle effect provides direct measurements of the magnetic vector in prominences (Leroy 1985, Bommier \& Leroy 1997). Such observations suggest that the field of most (but not all) prominences is relatively horizontal and has a polarity opposite to that indicated by photospheric magnetograms.

Spicules probably also are magnetic in nature, although the evidence in this case is more indirect. Models of spicules generally involve waves travelling along magnetic flux tubes. These locally "lift" the transition zone to greater heights thus producing a narrow extension of chromospheric material into the corona (Shibata 1982, Sterling \& Hollweg 1988).

Finally, there is growing evidence that explosive events are the signatures of small-scale reconnection, as proposed by Dere et al. (1991). Explosive events are identified as localized and short-lived intense broadenings of transition region spectral lines, sometimes in association with a line shift and often located near the magnetic network in the quiet Sun (Dere et al. 1989). Recently, raster scans carried out with SUMER onboard SOHO have yielded sets of spectra of explosive events that correspond to the expected signature of magnetic reconnection (Innes et al. 1996).

\section{Coronal manifestations}

Given the energetic dominance of the magnetic field in coronal layers it is not surprising that the field is directly or indirectly responsible for a large fraction of observed coronal phenomena. These include the temperature of the corona (see Ulmschneider, this volume), its large-scale structure (streamers, coronal holes, plumes), flares, coronal mass ejections and X-ray loops, jets and bright points. Space doesn't allow even a partial review of these phenomena and how they are thought to be related to the magnetic field. Let me just discuss the models for explaining two such phenomena.

The first applies to X-ray bright points, which are localized (6-9 $\mathrm{Mm}$ large) brightenings in $\mathrm{X}$-rays lasting 2 -48 hours and found all over the quiet parts of the solar disk. Priest et al. (1994) proposed a model for $\mathrm{X}$-ray bright points that is based on magnetic reconnection caused by the cancellation of two magnetic fragments of opposite polarity in the presence of an ambient magnetic field (magnetic canopy). This model agrees in a number of respects with observations of X-ray bright points, such as their association with cancelling magnetic features in the photosphere (Harvey 1985).

Another successful model is that for jets observed by the soft X-ray telescope on Yohkoh. These jets of X-ray bright material (i.e. collimated flows at temperatures of a few million degrees) shoot out from sites of particularly strong X-ray emission, often associated with smaller loop-like brightenings. Yokoyama \& Shibata (1995) presented simulations of the emergence of magnetic loops into a coronalike atmosphere filled with a magnetic field. Depending on whether this overlying field was inclined or horizontal they were able to reproduce one- or two-sided jets having properties similar to the observations.

The main direct source of information on the magnetic field in coronal layers is microwave emission. Above $4 \mathrm{GHz}$ the microwave radiation from active regions is dominated by gyroresonance opacity in strong coronal magnetic fields, which allows the strength of portions of the coronal field to be measured (see, e.g., Klein 1992 for a review).

Above a simple sunspot Lee et al. (1993) observed a coronal magnetic distribution compatible with a self-similar extrapolation of the photospheric distribution. Similarly, Schmelz et al. (1994) found that the field strengths deduced from microwave observations in parts of an active region were well reproduced by potential field extrapolations from the photosphere. At least in some active regions, however, microwave observations are providing evidence for significant currents, i.e. strongly non-potential fields (Alissandrakis et al. 1980, Lee et al. 1997).

\section{References}

Alissandrakis C.E., Kundu M.R., Lantos P., 1980, Astron. Astrophys. 82, 30-40. 
Bogdan T.J., Brown T.M., Lites B.W., Thomas J.H., 1993, A strophys. J. 406, 723-734.

Bommier V., Leroy J.L., 1997, in New Perspectives on Solar Prominences, D. Webb, B. Schmieder, D. Rust (Eds.), , IAU Coll. 167 in press

Braun D.C., Duvall T.L., Jr., La Bonte B.J., Jefferies S.M., Harvey J.W., Pomerantz M.A., 1992, A strophys. J. 391 , L113-L116.

Carlsson M., Stein R.F., 1995, Astrophys. J. 440, L29-L32.

Degenhardt D., Wiehr E., 1991, Astron. Astrophys. 252, 821-826.

Dere K.P., Bartoe J.-D.F., Brueckner G.E., 1989, Solar Phys. 123, 41-68.

Dere K.P., Bartoe J.-D.F., Brueckner G.E., Ewing J., Lund P., 1991, J. Geophys. Res. 96, 9399

Elsworth Y., Howe R., Isaak G.R., McLeod C.P., Miller B.A., New R., Speake C.C., Wheeler S.J., 1994, Astrophys. J. 434, 801-806.

Fontenla J.M., Avrett E.H., Loeser R., 1993, Astrophys. J. 406, 319-345.

Foukal P., Lean J., 1988, Astrophys. J. 328, 347-357.

Harvey K.L., 1985, Australian J. Phys. 38, 875

Harvey K.L., Harvey J.W., Martin S.F., 1975, Solar Phys. 40, 87-102.

Innes D.E., Inhester B., Axford W.I., Wilhelm K., 1997, Nature 386, 811-813.

Keller C.U., Deubner F.-L., Egger U., Fleck B., Povel P., 1994, Astron. Astrophys. 286, 626-634.

Klein L., 1992, in Methods of Solar and Stellar Magnetic Field Determination, M. Faurobert-Scholl, N. Mein, H. Frisch (Eds.), Obs. de Paris, Meudon, p. 113-130.

Lee J.W., Hurford G.J., Gary D.E., 1993, in The Magnetic and Velocity Fields of Solar Active Regions, H. Zirin , G. Ai, H. Wang (Eds.), Astron. Soc. Pacific Conf. Series, IA U Coll. 141, 287-290.

Lee J.W., White S.M., Gopalswamy N., Kundu M.R., 1997, Solar Phys. submitted

Libbrecht K.G., Woodard M.F., 1990, Nature 345, 779-782

Lin H., 1995, Astrophys. J. 446, 421 430

Leroy J.L., 1985, in Measurements of Solar Vector Magnetic Fields, M.J. Hagyard (Ed.), NASA Conf. Publ. 2374, p. $121-140$.

Martin S.F., 1988, Solar Phys. 117, 243-259.

Parker E.N., 1988, Astrophys. J. 330, 474-479.

Priest E.R., Parnell C.E., Martin S.F., 1994, Astrophys. J. 427, 459-474.

Schmelz J.T, Holman G.D., Brosius J.W., Willson R.F., 1994, Astrophys. J. 434, 786-794.

Schrijver C.J., Coté J., Zwaan C., Saar S.H., 1989, Astrophys. J. 337, 964-976.

Shibata K.: 1982, Solar Phys. 81, 9-17.

Solanki S.K., Schmidt H.U., 1993, A stron. Astrophys. 267, 287-291.

Solanki S.K., Unruh Y., 1997, Astron. Astrophys. in press

Solanki S.K., Livingston W., Ayres T., 1994, Science 263, 64-66.

Solanki S.K., Zuffrey D., Lin H., Rüedi I., Kuhn J., 1996, Astron. Astrophys. 310, L33-L36.

Spruit H.C., 1982, Astron. Astrophys. 108, 348-355.

Sterling A.C., Hollweg J.V., 1988, A strophys. J. 327, 950-963.

Tarbell T., Peri M., Frank Z., Shine R., Title A., 1988, Seismology of the Sun and Sun-like Stars, ESA SP-286, p. 315-319.

Title A., 1997, in Cool Stars, Stellar Systems and the Sun, X, J.A. Bookbinder, R. Donahue (Eds.), Harvard Smithsonian Center for Astrophysics, Cambridge, MA, in press

Title A.M., Frank Z.A., Shine R.A., Tarbell T.D., Topka K.P., Scharmer G., Schmidt W., 1993, Astrophys. J. 403, 780-796.

Venkatakriohnan P., 1986, Nature 322, 156-157.

Willson R.C., Hudson H.S., 1991, Nature 351, 42-44.

Yokoyama T., Shibata K., 1995, Nature 375, 42-44.

Zirin H., 1987, Solar Phys. 110, 101-107. 